\title{
All-Epiphyseal Physeal-Sparing Anterior Cruciate Ligament Reconstructive Surgery: A Study of 3-Dimensional Modeling to Characterize a Safe and Reproducible Surgical Approach
}

\author{
Daniel A. Marchwiany, M.D., Cody Lee, M.D., Philip Ghobrial, B.S., Richard Lawley, M.D., \\ and Steven C. Chudik, M.D.
}

Purpose: The purpose of this study was to use 3-dimensional magnetic resonance imaging modeling of the skeletally immature knee to help characterize safe and reproducible tunnel positions, diameters, lengths, trajectories, and distances from anatomic landmarks and the physeal and articular cartilage for physeal-sparing anterior cruciate ligament (ACL) reconstructive surgery. Methods: Magnetic resonance imaging from 19 skeletally immature knees with normal anatomy were gathered. The 3-dimensional models were created, and the relevant anatomic structures were identified. Cylinders simulating tunnel length, diameter and trajectory were superimposed onto the models, and descriptive measurements were performed. Results: A safe position for the creation of an $8 \mathrm{~mm}$ diameter femoral tunnel was described in the lateral femoral condyle. The femoral tunnel length averaged $25.5 \pm 2.6 \mathrm{~mm}$. The bony entry point was located $3.8 \pm 2.4 \mathrm{~mm}$ proximally and $12.7 \pm 2.2 \mathrm{~mm}$ posteriorly to the lateral epicondyle. The shortest distance from the tunnel edge to the physis and femoral articular cartilage was $2.8 \pm 0.7 \mathrm{~mm}$ and $3.7 \pm 0.9 \mathrm{~mm}$, respectively. The safe position for an $8 \mathrm{~mm}$ diameter tibial tunnel was also identified and described in the proximal tibia. The epiphyseal tibial tunnel length from the ACL footprint to the physis averaged $15.5 \pm 1.6 \mathrm{~mm}$. The proximal tibial epiphysis was found to accommodate a tibial crosspin measuring $63.5 \pm 5.9 \mathrm{~mm}$ in length and $8.2 \pm 1.5 \mathrm{~mm}$ in diameter without disrupting the physis or articular cartilage. Conclusions: Three-dimensional modeling created from magnetic resonance imaging can help define important anatomic relationships for physeal-sparing ACL reconstructive surgery in skeletally immature knees and may assist in reducing the risk of injury to local anatomic structures. Clinical Relevance: Knowledge of the anatomic relationships in skeletally immature knees serves as a valuable reference for surgeons performing physeal-sparing ACL reconstruction surgery.

$S^{n}$ urgical reconstruction of the anterior cruciate ligament (ACL) is the standard of care for the skeletally immature patient because nonoperative treatment has been associated with persistent knee instability, secondary injury to the meniscus, and articular cartilage, osteoarthritis and poor long-term outcomes. ${ }^{1-3}$ Traditional ACL reconstructive techniques use transphyseal tunnels drilled through the physes of the distal femur

From the Department of Orthopaedics, University of North Carolina Medical Center, Chapel Hill, North Carolina, U.S.A. (D.A.M.); Pritzker School of Medicine at University of Chicago, Chicago, Illinois, U.S.A. (C.L.); Loyola University Chicago Stritch School of Medicine, Maywood, Illinois, U.S.A. (P.G.); Cornerstone Orthopedics, Wheat Ridge, Colorado, U.S.A. (R.L.); Hinsdale Orthopaedics, Westmont, Illinois, U.S.A. (S.C.C.); and Orthopaedic Surgery and Sports Medicine Teaching and Research Foundation, Westmont, Illinois, U.S.A. (S.C.C.).

The authors report no conflicts of interest in the authorship and publication of this article. Full ICMJE author disclosure forms are available for this article online, as supplementary material. and proximal tibia, which can potentially manifest in angular and longitudinal growth abnormalities. ${ }^{3-7}$ Alternative techniques have been developed to minimize injury by not traversing the physis with tunnels, fixation hardware or the ACL graft. ${ }^{7-13}$ These physealsparing techniques for ACL reconstruction are performed less commonly and are more technically challenging, placing the physis, articular cartilage and

Received September 14, 2019; revised manuscript received February 3, 2020; accepted April 2, 2020.

Address correspondence to Daniel A Marchwiany, M.D., 1919 So-Hi Drive, Durham, NC 27703,U.S.A.E-mail:dmarchwiany@gmail.com

(C) 2020 THE AUTHORS. Published by Elsevier Inc. on behalf of the Arthroscopy Association of North America. This is an open access article under the CC BY-NC-ND license (http://creativecommons.org/licenses/by-nc-nd/4.0/). 2666-061X/191089

https://doi.org/10.1016/j.asmr.2020.04.002 
other anatomic structures at an increased risk for inadvertent injury. ${ }^{14}$ Our understanding of safe positions for physeal-sparing tunnels for physeal-sparing ACL reconstruction techniques is limited. Therefore, characterization of safe positions for these physeal-sparing tunnels would be helpful to surgeons to improve the safety and reproducibility of these techniques.

The senior author's (SCC) preferred technique for physeal-sparing ACL reconstruction is modeled in this study. The technique is performed with arthroscopic and fluoroscopic guidance. The tibial tunnel starting point is identified arthroscopically as the anatomic ACL footprint and is drilled antegrade with a retrocutter over a guidepin that exits distally at the anterior tibial cortex. Fluoroscopy is used to guide the depth of the tunnel so as to avoid violating the physis. The guidepin is retained and is next used for the tibial crosspin guide to place a second guidepin medial-to-lateral across the entire epiphysis and centrally through the tibial tunnel. This guidepin is exchanged for a passing wire. The femoral tunnel is drilled with an outside-in femoral ACL guide. The tip is placed at femoral origin site, and a guidewire is placed from the lateral femoral cortex epiphysis of the femur to that site. Fluoroscopy is used to confirm that the guidepin does not violate the physis, and then it is over-reamed with cannulated reamers to the appropriate diameter. A loop-ended soft-tissue graft is passed using the passing wire outside-in from the femoral tunnel to the tibial tunnel. The looped end of the graft is secured by a tibial crosspin placed medial to lateral over the passing wire. The femoral side of the graft is secured by an interference screw. The graft, hardware and graft tunnels all remain entirely epiphyseal.

Magnetic resonance imaging (MRI) imaging and computer modeling have been used to identify and define pertinent knee anatomy involved with surgical ligament reconstructive techniques. ${ }^{15-18}$ The purpose of this study was to use 3-dimensional magnetic resonance imaging (MRI) modeling of the skeletally immature knee to help characterize safe and reproducible tunnel positions, diameters, lengths, trajectories, and distances from anatomic landmarks and the physeal and articular cartilage for physeal-sparing ACL reconstructive surgery. We hypothesized that data gathered through 3-dimensional modeling will help to reveal safe and reproducible positions for these tunnels relative to important anatomic landmarks, ultimately providing the potential for safer execution of physealsparing ACL surgery techniques.

\section{Methods}

Following an Institutional Review Board -approved protocol, medical records from the senior author's practice were retrospectively reviewed to identify all skeletally immature patients who had undergone dedicated knee MRI during the previous 12 months.
Patients were excluded if they had past histories or radiologic evidence of congenital, developmental or acquired anatomic abnormalities. Each MRI was assessed by the senior author, a board-certified orthopaedic surgeon who is fellowship-trained in sports medicine. MRI images were scanned at 1.5 Tesla with multiplanar 2D proton density and Tl-weighted imaging sequences. Image files were uploaded with Mimics (version 16.0; Materialise, Leuven, Belgium) and 3-matic (version 8.0; Materialise) computer software to create 3-dimensional models of the knee in extension so as to simulate surgical tunnels and to measure pertinent anatomic relationships (Fig 1).

A complete mask of the bone and physes was created by manually outlining each of the structures from the sagittal images to minimize errors in defining borders and maximizing accuracy of the 3-dimensional models. Relevant bony landmarks, tendinous insertion sites and ligamentous footprints were identified, and their borders were defined in the sagittal, coronal and axial image sets and marked on the 3-dimensional computer models. More specifically, we mapped and marked the location of the femoral epicondyle and the femoral footprints of the ACL, fibular collateral ligament (FCL) and popliteus tendon. On the proximal tibia, we mapped and marked the location of the tibial tubercle and the tibial footprints of the ACL, the patellar tendon and the pes anserine. For the purpose of measuring the relative distances, the tibial tubercle and lateral femoral epicondyle were defined by single points corresponding to their most prominent and palpable aspects. The ACL footprints on both the femur and tibia were first outlined on cross-sectional imaging. An average was calculated of these points to determine the center of the ACL footprint to be used for tunnel measurements.

Cylinders simulating the epiphyseal tunnels were superimposed into the 3-dimensional models (Fig 1). An $8 \mathrm{~mm}$ diameter cylinder was selected to simulate a femoral tunnel, which would accommodate the lower limit in size for an autograft pediatric hamstring graft. The starting point of the femoral tunnel was measured to be the center of a "safe window," defined as a circle bordered by the femoral physis superiorly, the femoral articular cartilage posteriorly and the femoral footprint of the FCL anteriorly (Fig 2). An $8 \mathrm{~mm}$ diameter cylinder simulating the graft tunnel was superimposed from the center of the safe window to the center of the femoral ACL footprint. We measured the tunnel length, the tunnel entry window diameter, the distance from the lateral epicondyle to the tunnel center on the lateral femoral cortex, the angle of the femoral tunnel relative to a line perpendicular to the longitudinal anatomic femoral axis in the coronal and sagittal planes, and the shortest distance from the tunnel perimeter to the physis and articular cartilage. The position of the tunnel's bony entry point was identified on the lateral 


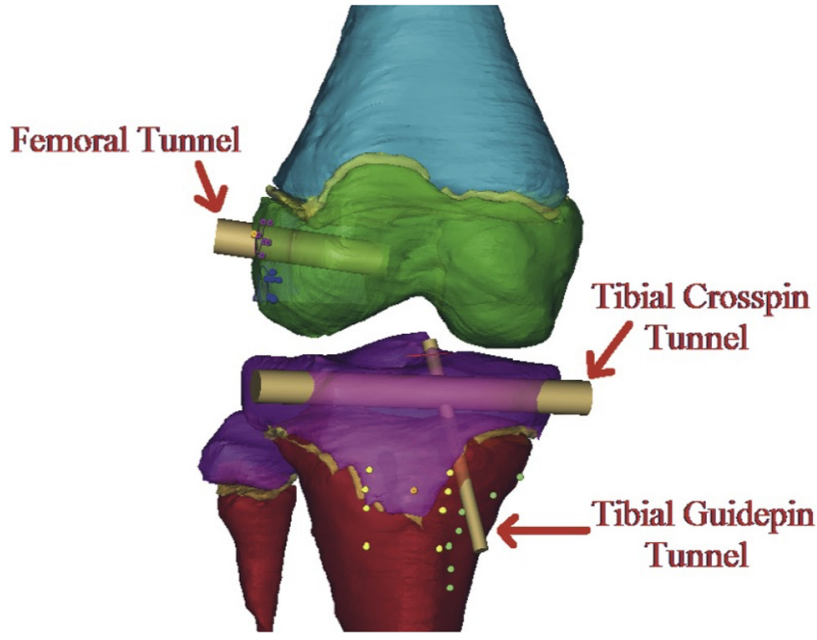

Fig 1. Anterior view of a 3-dimensional model of a skeletally immature right knee. This model displays the distal femur and proximal tibia with mapped landmarks and superimposed surgical tunnels. The mask of articular cartilage is subtracted in this figure.

femoral cortex by measuring from the center point of the tunnel to the lateral femoral epicondyle (Fig 3).

An $8 \mathrm{~mm}$ cylinder was positioned in the proximal tibial epiphysis coursing along a central axis from the center of the tibial ACL footprint toward a point on the anterior tibial cortex $1 \mathrm{~cm}$ proximal to the tibial physis and equidistant between the medial edge of the patellar tendon footprint and the superior-lateral edge of the pes anserine. We measured the distance from the tibial ACL footprint to the anterior tibial cortex, representing the path of the tibial guidewire; the tunnel length from the ACL footprint to the physis, representing the maximum depth of tibial graft tunnel; the distance from the apophysis of the tibial tubercle to the tunnel entry center; and the tunnel angle relative to the plane of the tibial plateau. The position of the tibial guidepin's bony entry point was identified on the anterior tibial cortex by measuring the distance from the tibial guidepin's starting point to the tibial tubercle (Fig 4).

Further, to provide for an alternative method of all-epiphyseal fixation, a cylinder, representing a tibial epiphyseal crosspin, was placed parallel in the axial plane to the posterior surface of the femoral condyles and intersecting the center of the tibial cylinder. To assess the available space for a tibial epiphyseal crosspin to secure a looped end of a soft-tissue graft, the length and diameter were measured for the largest cylinder possible that did not contact either the physis inferiorly or the articular cartilage superiorly. Last, our 3-dimensional models were created using MRIs of knees in full extension. To help predict how traversing tendons may narrow the femoral safe window as the knee is flexed, we simulated knee flexion with our model by creating an outline of the popliteus tendon and FCL and rotating these in the sagittal plane around the insertion point on the femoral condyle to see at what angle these structures would intersect the femoral safe window.

All measurements were performed 3 times by a trained member of the research team. The average of the measurements was used for our analysis.

\section{Statistical Analysis}

Basic descriptive statistics were performed using SPSS 21.0 (IBM, Armonk, NY, USA). Mean values were reported with standard deviations, confidence intervals and ranges.

\section{Results}

Following review, we obtained a total of 19 MRIs from healthy skeletally immature patients (11 males and 8 females) with a mean age of 12.2 years (range 8-15 years). The lateral femoral condylar epiphysis allowed a maximum tunnel diameter of $12.1 \pm 1.5 \mathrm{~mm}$ from the determined bony entry point on the lateral cortex to the femoral ACL footprint without injuring the physeal or articular cartilage (Table 1). The femoral tunnel length was $25.5 \pm 2.6 \mathrm{~mm}$. The tunnel entered the lateral femoral cortex centered in the safe window at a point located $3.8 \pm 2.4 \mathrm{~mm}$ proximally and $12.7 \pm 2.2 \mathrm{~mm}$ posteriorly to the lateral epicondyle with the physis above, the articular cartilage posterior and the FCL anterior. The tunnel coursed lateral to medial, nearly perpendicular to the longitudinal axis of the femur in the coronal plane. It coursed at an angle of $10.5^{\circ} \pm 5.0^{\circ}$ distally in the coronal plane and at an angle of $6.4^{\circ} \pm 5.7^{\circ}$ anteriorly in the axial plane, both relative to a line perpendicular to the anatomic longitudinal axis of the femur. The shortest distance from the tunnel perimeter to the physis was $2.8 \pm 0.7 \mathrm{~mm}$ and to the femoral articular cartilage, it was $3.7 \pm 0.9 \mathrm{~mm}$. Manipulation of the 3-dimensional models revealed that the safe window in the lateral femoral cortex was maintained between $0^{\circ}$ and $60^{\circ}$ of knee flexion but is reduced at greater angles of flexion.

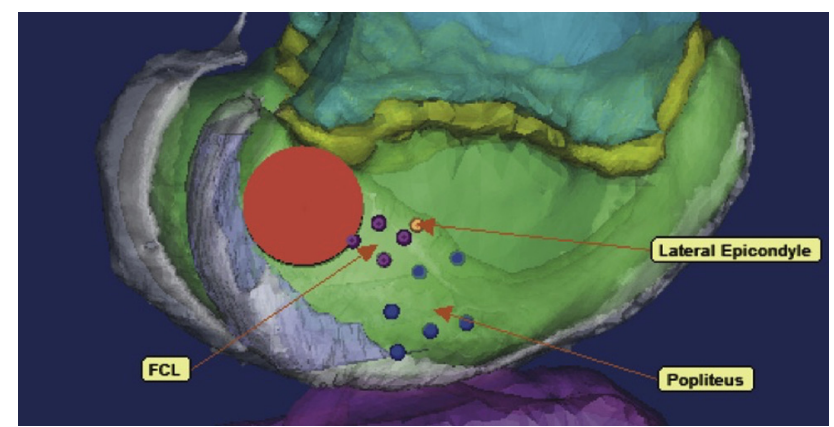

Fig 2. Sagittal view of the left, lateral femoral condyle showing the femoral tunnel window (red circle) defined by its bordering landmarks: the femoral physis proximally, the articular cartilage posteriorly and the fibular collateral ligament anteriorly. The purple dots represent the origin of the FCL; the blue dots represent the popliteus insertion site. 


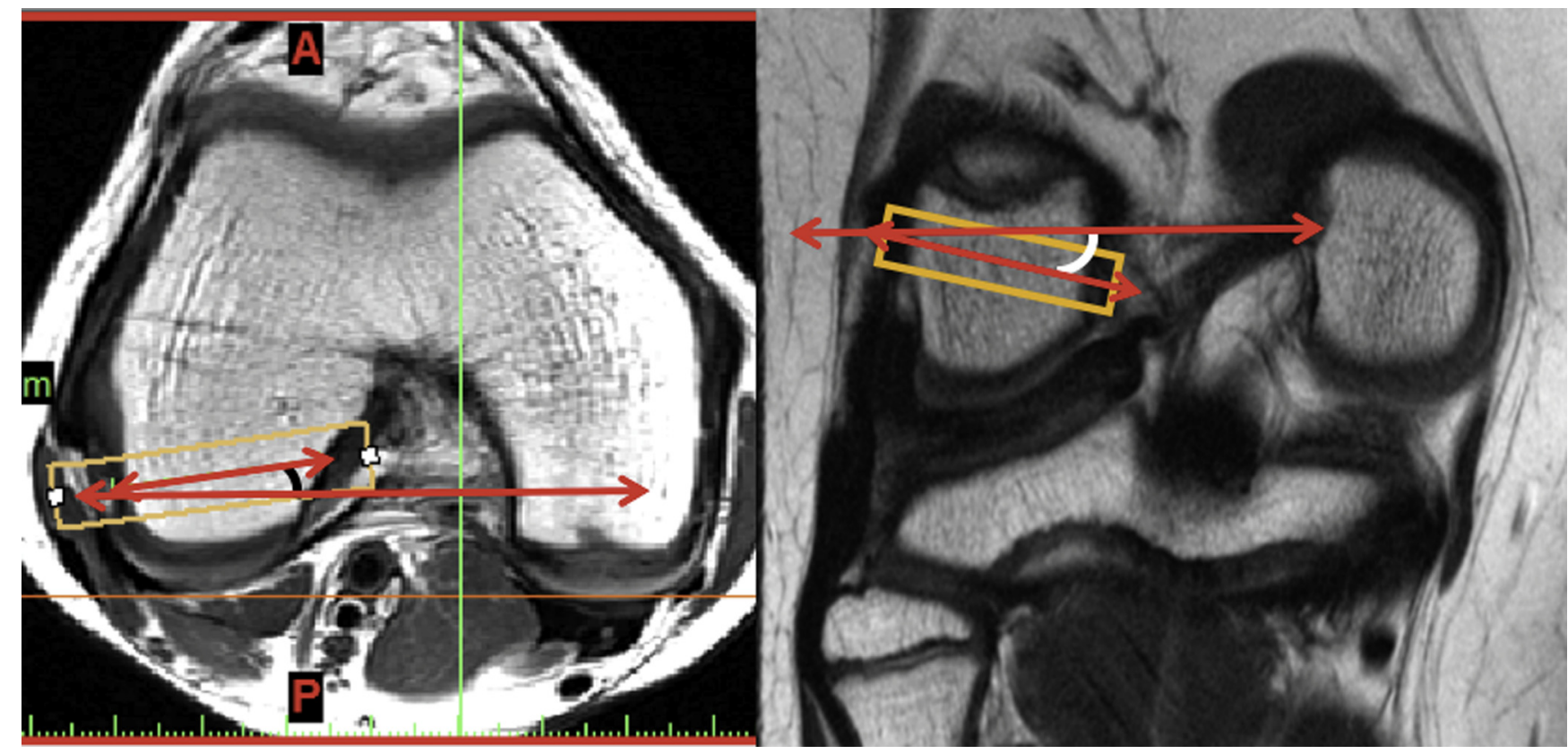

Fig 3. Once tunnels were superimposed onto the 3D models, measurements were taken from the MRIs to determine the tunnel lengths, trajectories and diameters. The tunnel trajectory of the femoral tunnel is being measured in the axial (left) and coronal (right) planes. The long, horizontal arrow in each image depicts the horizontal plane of the femoral epiphysis.

The tibial epiphysis allowed a tunnel length from the ACL footprint to the physis of $15.5 \pm 1.6 \mathrm{~mm}$ (Table 2). To create the tibial tunnel, a small-diameter guide pin entered the anterior tibial cortex centered on a point located $14.1 \pm 2.4 \mathrm{~mm}$ medially and $0.1 \pm 3.8 \mathrm{~mm}$ distally from the tibial tubercle, and $34.3 \pm 3.0 \mathrm{~mm}$ from the tibial ACL footprint. The angle of the tunnel from the entry point to the ACL footprint was measured as $65.2^{\circ} \pm 5.7^{\circ}$ relative to the plane of the tibial plateau. The tibial epiphysis width at the intersection of the epiphyseal tunnel and parallel to the posterior femoral condyles measured $63.5 \pm 5.9 \mathrm{~mm}$ and allowed a maximum tunnel diameter of $8.2 \pm 0.8 \mathrm{~mm}$ without violating either the physeal or articular cartilage (Table 3).

\section{Discussion}

The data yielded by our study describes safe positions to create femoral and tibial epiphyseal tunnels from superficial bony landmarks and angles of trajectory for physeal-sparing ACL reconstructive surgery. For the femoral epiphyseal tunnel, the safe position was located at the lateral femoral cortex $3.8 \pm 2.4 \mathrm{~mm}$ proximally and $12.7 \pm 2.2 \mathrm{~mm}$ posteriorly to the lateral epicondyle toward the ACL footprint at an angle of $10.5^{\circ} \pm 5.0^{\circ}$ distally and an angle of $6.4^{\circ} \pm 5.7^{\circ}$ anteriorly from a line perpendicular to the longitudinal axis of the femur. For the tibial epiphyseal tunnel, the safe position was located at the the anterior tibial cortex $14.1 \pm 2.4 \mathrm{~mm}$ medially and $0.1 \pm 3.8 \mathrm{~mm}$ distally from the tibial tubercle toward the ACL footprint at an angle $65.2^{\circ} \pm 5.7^{\circ}$ relative to the plane of the tibial plateau. These data may serve as a foundation for future research to establish consistent and safe tunnel placement for physeal-sparing ACL reconstructive surgery. Further, this information may serve to assist surgeons while they continue to perform these procedures with point-to-point guides under fluoroscopic and arthroscopic guidance.

Other studies have examined the length and relative position of the femoral epiphyseal tunnel. From our determined safe starting point, the femoral epiphyseal tunnel length measured $25.5 \pm 2.6 \mathrm{~mm}$. Xerogeanes et al. reported that for a femoral tunnel traversing the

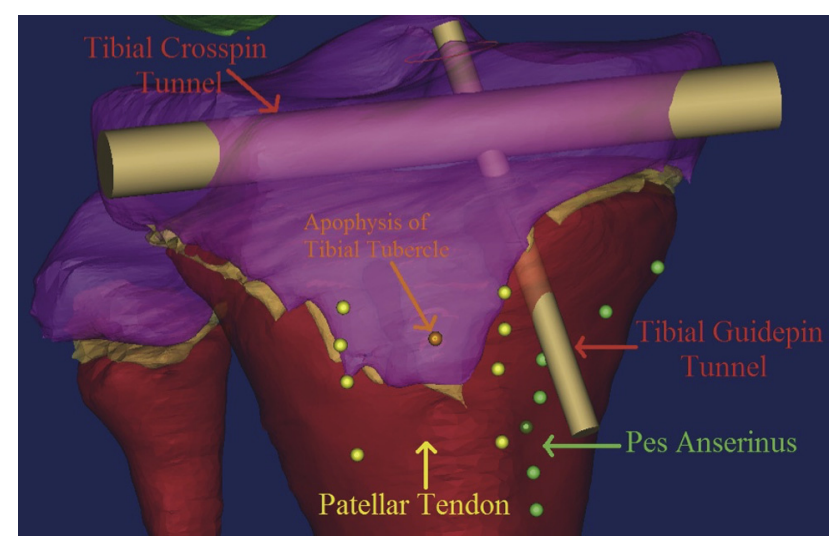

Fig 4. Anterior view of the right tibia with pertinent landmarks and surgical tunnels superimposed onto the 3D model. Yellow dots represent the medial and lateral borders of the patellar tendon. Green dots represent the lateral border of the pes anserinus. 
Table 1. Anatomic Measurements for Femoral Epiphyseal Tunnel

\begin{tabular}{|c|c|c|c|}
\hline & Mean \pm SD & $\begin{array}{c}\text { Confidence } \\
\text { Intervals }(95 \%)\end{array}$ & Range \\
\hline Tunnel length (mm) & $25.5 \pm 2.6$ & $24.3-26.7$ & $20.6-30.4$ \\
\hline Tunnel entry window diameter (mm) & $12.1 \pm 1.5$ & 11.512 .8 & $9.8,14.8$ \\
\hline Distance from lateral epicondyle to tunnel center (proximal) (mm) & $3.8 \pm 2.4$ & 2.84 .9 & $-1.2-8.6$ \\
\hline Distance from lateral epicondyle to tunnel center (posterior) (mm) & $12.7 \pm 2.2$ & 11.713 .7 & $8.1,15.9$ \\
\hline $\begin{array}{l}\text { Angle of femoral tunnel relative to line perpendicular to longitudinal } \\
\text { femoral axis in coronal plane (degrees; distally) }\end{array}$ & $10.5 \pm 5.0$ & 8.212 .7 & $4.0-20.1$ \\
\hline $\begin{array}{l}\text { Angle of femoral tunnel relative to line perpendicular to longitudinal } \\
\text { femoral axis in sagittal plane (degrees; anteriorly)* }\end{array}$ & $6.4 \pm 5.7$ & 3.99 .0 & $-4.2-17.5$ \\
\hline $\begin{array}{l}\text { Shortest distance from tunnel edge to physis with } 8 \mathrm{~mm} \text { diameter } \\
\text { tunnel }(\mathrm{mm})\end{array}$ & $2.8 \pm 0.7$ & $2.5-3.1$ & $1.1-4.0$ \\
\hline $\begin{array}{l}\text { Shortest distance from tunnel edge to articular cartilage with } 8 \mathrm{~mm} \\
\text { diameter tunnel }(\mathrm{mm})\end{array}$ & $3.7 \pm 0.9$ & 3.3-4.1 & $1.7-5.3$ \\
\hline
\end{tabular}

*Positive values represent a tunnel coursing anteriorly toward the ACL footprint.

space between the ACL femoral footprint and the insertion of the popliteus, its length ranged from 27.93 to $30.19 \mathrm{~mm}$ in children ages 7 to 17 . Further, a study by Davis et al. found a lateral femoral condylar width of $24.76 \pm 2.71 \mathrm{~mm}$ in children ages 7 to 12 and $26.22 \pm$ $3.11 \mathrm{~mm}$ in children ages 12 to $16 .^{12,19}$ Our measurements are consistent with the data reported by these studies, reaffirming the expected length and relative position of the femoral epiphyseal tunnel.

By simulating an $8 \mathrm{~mm}$ diameter femoral tunnel positioned in our safe window, it was determined that the shortest distance from the perimeter of the tunnel to the physis $(2.8 \pm 0.7 \mathrm{~mm})$ and to the articular cartilage $(3.7 \pm 0.9 \mathrm{~mm})$ occurred at the lateral cortex of the femoral condyle. Xerogeanes et al. reported that the distance from the center of the tunnel to the physis ranged from 11.25 to $12.06 \mathrm{~mm}$, and the distance to the articular cartilage ranged from 5.06 to $10.63 \mathrm{~mm}^{.12}$ Differences in measured distances from the articular and physeal cartilage are the result of differences in tunnel position. The tunnel simulated by Xerogeanes et al. coursed from the popliteus insertion site on the lateral femoral condyle to the ACL footprint, whereas our simulated tunnel coursed from a point on the lateral femoral cortex centered in a defined safe window, avoiding injury to important anatomic structures, including the popliteus tendon and the fibular collateral ligament. Davis et al. measured epiphyseal dimensions and did not simulate tunnels. Xerogeanes et al., on the other hand, studied femoral tunnels but did not model tibial tunnels. In our study, we simulated both tibial and femoral tunnels to provide a quantified description of safe surgical femoral and tibial tunnel positions for the purpose of developing standardized methods for physeal-sparing ACL reconstruction.

Our findings are consistent with other studies that measured the dimensions of the tibial epiphysis. Our study discovered that the tibial epiphyseal tunnel length from the ACL footprint to the physis is $15.5 \pm 1.6 \mathrm{~mm}$, coursing at an angle of $65.2^{\circ} \pm 5.7^{\circ}$ relative to the plane of the tibial plateau. Davis et al. reported a vertical epiphyseal height of $15.26 \pm 1.25 \mathrm{~mm}$ in children ages 7 to 12 years and $15.01 \pm 2.14 \mathrm{~mm}$ in children ages 12 to 16 years, and Swami et al. reported $14.8 \pm 1.2 \mathrm{~mm}$ in 6 -year-old patients to $16.6 \pm 1.6 \mathrm{~mm}$ in 15 -year-old patients. $^{19,20}$

Because of challenges with anatomic ACL reconstruction in skeletally immature patients, some described techniques cross the tibial physis with the graft or suture and fix the graft in or on the metaphysis of the tibia across the proximal tibial physis. ${ }^{2,9-11,21}$ To avoid crossing either the femoral or tibial physis with graft, hardware or graft tunnels, the senior author has been performing physeal-sparing ACL reconstruction with an autologous gracillis/semitendinosis hamstring graft using a crosspin to fix the looped end of the graft in the tibial epiphysis and an interference screw to fix the free end of the graft in the femoral epiphysis

Table 2. Anatomic Measurements for Tibial Epiphyseal Tunnel

\begin{tabular}{lrr}
\hline & & \multicolumn{1}{c}{ Confidence } \\
Intervals (95\%) & Mean \pm SD & $32.9-35.6$ \\
\hline Distance from tibial ACL footprint to anterior tibial cortex (mm) & $34.4 \pm 3.0$ & $14.8-16.3$ \\
Tunnel length from ACL footprint to physis (mm) & $15.5 \pm 1.6$ & $13.0-15.2$ \\
$\begin{array}{l}\text { Distance from the apophysis of the tibial tubercle to tunnel entry } \\
\quad \text { center (medial) (mm) }\end{array}$ & $14.1 \pm 2.4$ & $-1.6-1.9$ \\
$\begin{array}{l}\text { Distance from the apophysis of the tibial tubercle to tunnel entry } \\
\quad \text { center (distal) (mm) }\end{array}$ & $0.1 \pm 3.8$ & $-6.3-5.9$ \\
$\quad$ Tunnel angle relative to the tibial plateau (degrees) & $65.2 \pm 5.7$ & $62.7-67.8$ \\
\hline
\end{tabular}


Table 3. Anatomic Measurements for Tibial Epiphyseal Fixation

\begin{tabular}{lccc}
\hline & Mean \pm SD & Confidence Intervals (95\%) & Range \\
\hline Tibial epiphyseal width $(\mathrm{mm})$ & $63.5 \pm 5.9$ & $60.8-66.1$ & $52.6-73.5$ \\
Maximal tunnel diameter within the epiphysis $(\mathrm{mm})$ & $8.2 \pm 1.5$ & $7.5-8.9$ & $5.4-11.2$ \\
\hline
\end{tabular}

(Fig 5) (Video 1). In this study, we discovered that the proximal tibial epiphysis, even with undulations, allows for a tibial crosspin measuring up to $63.5 \pm 5.9$ $\mathrm{mm}$ in length and up to $8.2 \pm 0.8 \mathrm{~mm}$ in diameter without breaching either the physis or the articular cartilage.
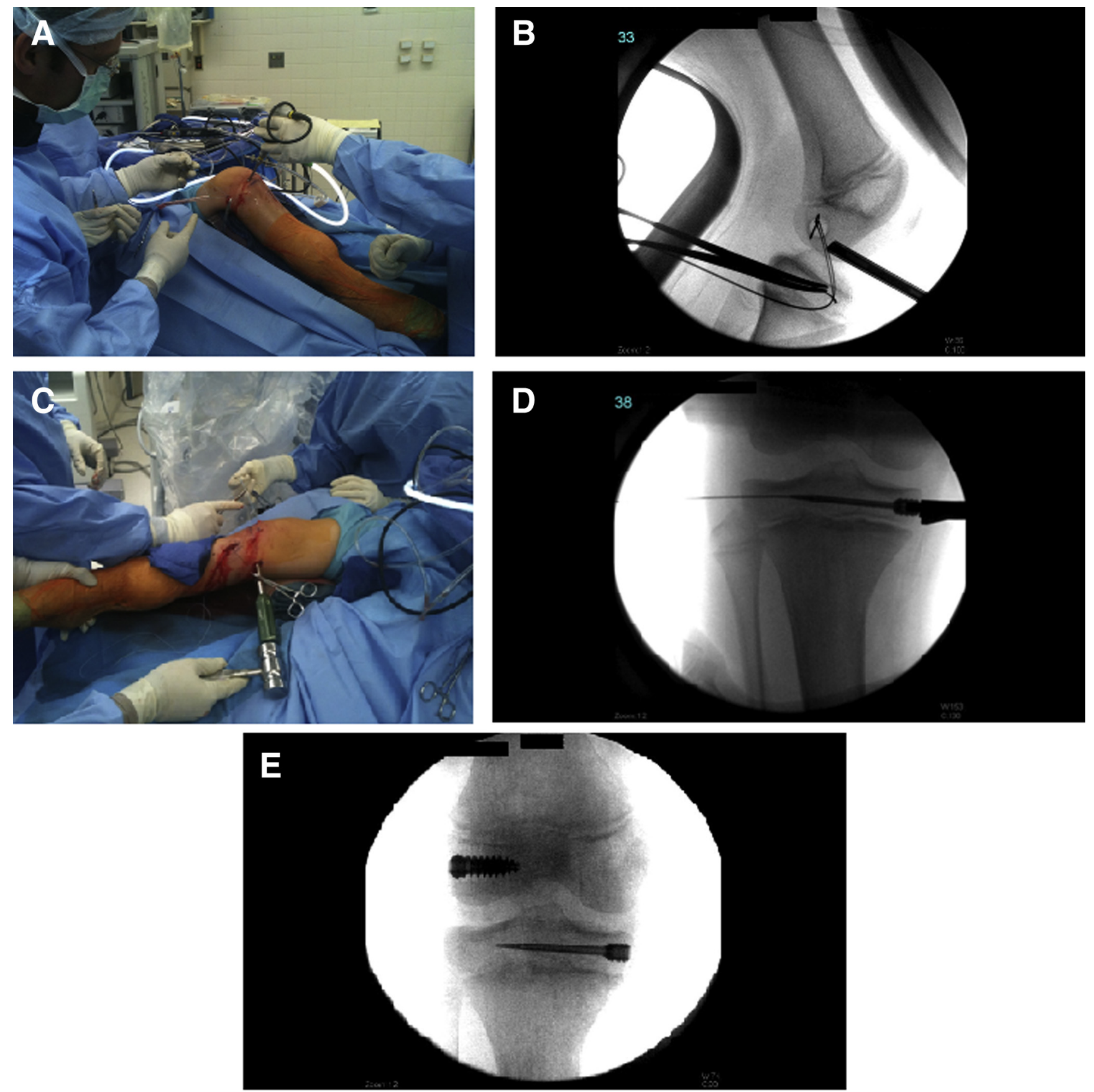

Fig 5. (A) Fluoroscopic lateral view of the right knee showing the femoral and tibial tunnels. (B) The tibial crosspin is placed to secure the graft within the tibial epiphysis. (C) Fluoroscopic anteroposterior image that demonstrates final hardware positioning. 


\section{Limitations}

There are some limitations present in the methods of our study. For instance, this study used MRI with a slice thickness of up to $3.5 \mathrm{~mm}$ in the sagittal plane, which can limit the resolution and accuracy of the 3-dimensional models. To minimize this effect, all 3 MRI cross-sections (axial, coronal and sagittal) were used during structure localization to define most accurately the landmarks and other structures; however, some data were likely lost despite using this method. Further, it is possible that variations in the dimensions or size of the knee may influence the location of the safe window defined in the present study. We did not account for such variations in our study design, which may serve as another potential limitation to our study. Another limitation is that there was only a single investigator, so we are not able to provide intra- and interobserver reliability data. Last, our 3-dimensional models were created from MRIs taken of patients with their knees in full extension. Knee position during ACL reconstruction varies depending on the technique; degree of flexion may range from $20^{\circ}$ to $90^{\circ}$, depending on the preference of the surgeon. ${ }^{22-24}$ Flexing the knee changes the relative position of the FCL and popliteus tendon along the femur, which can narrow the femoral safe window defined in our study. Therefore, if drilling the femoral epiphyseal tunnel in accordance with the parameters defined in this study, the angle of knee flexion must be less than $60^{\circ}$ to maintain the window's viability.

\section{Conclusions}

Three-dimensional modeling created from MRI can help to define important anatomic relationships for physeal-sparing ACL reconstructive surgery in skeletally immature knees and may assist in reducing the risk of injury to local anatomical structures.

\section{References}

1. Ramski DE, Kanj WW, Franklin CC, Baldwin KD, Ganley TJ. Anterior cruciate ligament tears in children and adolescents: A meta-analysis of nonoperative versus operative treatment. Am J Sports Med 2014;42:2769-2776.

2. Vavken P, Murray MM. Treating anterior cruciate ligament tears in skeletally immature patients. Arthroscopy 2011;27:704-716.

3. Collins MJ, Arns TA, Leroux T. Growth abnormalities following anterior cruciate ligament reconstruction in the skeletally immature patient: A systematic review. Arthroscopy 2016;32:1714-1723.

4. Larson CM, Heikes CS, Ellingson CI, Wulf CA, Giveans MR, Stone RM, et al. Allograft and autograft transphyseal anterior cruciate ligament reconstruction in skeletally immature patients: Outcomes and complications. Arthroscopy 2016;32:860-867.

5. Koman JD, Sanders JO. Valgus deformity after reconstruction of the anterior cruciate ligament in a skeletally immature patient: A case report. J Bone Joint Surg Am 1999;81:711-715.
6. Seil R, Pape D, Kohn D. The risk of growth changes during transphyseal drilling in sheep with open physes. Arthroscopy 2008;24:824-833.

7. Fabricant PD, Jones KJ, Delos D, Cordasco FA, Marx RG, Green DW, et al. Reconstruction of the anterior cruciate ligament in the skeletally immature athlete: A review of current concepts: AAOS exhibit selection. J Bone Joint Surg Am 2013;95:e28.

8. Anderson AF. Transepiphyseal replacement of the anterior cruciate ligament using quadruple hamstring grafts in skeletally immature patients. J Bone Joint Surg Am 2004;: 201-209 (Pt 2):86-A Suppl 1.

9. Kocher MS, Heyworth BE, Fabricant PD, Tepolt FA, Micheli LJ. Outcomes of physeal-sparing acl reconstruction with iliotibial band autograft in skeletally immature prepubescent children. J Bone Joint Surg Am 2018;100: 1087-1094.

10. Lykissas MG, Nathan ST, Wall EJ. All-epiphyseal anterior cruciate ligament reconstruction in skeletally immature patients: A surgical technique using a split tibial tunnel. Arthrosc Tech 2012;1:el33-el39.

11. Wong ES, Feeley BT, Pandya NK. Comparing outcomes between the over-the-top and all-epiphyseal techniques for physeal-sparing ACL reconstruction: A narrative review. Orthop J Sports Med 2019;7:2325967119833689.

12. Xerogeanes JW, Hammond KE, Todd DC. Anatomic landmarks utilized for physeal-sparing, anatomic anterior cruciate ligament reconstruction: An MRI-based study. J Bone Joint Surg Am 2012;94:268-276.

13. McCarthy MM, Graziano J, Green DW, Cordasco FA. Allepiphyseal, all-inside anterior cruciate ligament reconstruction technique for skeletally immature patients. Arthrosc Tech 2015;23:1206-1214.

14. Frosch KH, Stengel D, Brodhun T, Stietencron I, Holsten D, Hertel P, et al. Outcomes and risks of operative treatment of rupture of the anterior cruciate ligament in children and adolescents. Arthroscopy 2010;26:1539-1550.

15. Pietrini SD, LaPrade RF, Griffith CJ, Wijdicks CA, Ziegler CG. Radiographic identification of the primary posterolateral knee structures. Am J Sports Med 2009;37: 542-551.

16. Shea KG, Apel PJ, Pfeiffer RP, Showalter LD, Traughber PD. The tibial attachment of the anterior cruciate ligament in children and adolescents: Analysis of magnetic resonance imaging. Knee Surg Sports Traumatol Arthrosc 2002;10:102-108.

17. Shea KG, Grimm NL, Belzer JS. Volumetric injury of the distal femoral physis during double-bundle ACL reconstruction in children: A three-dimensional study with use of magnetic resonance imaging. J Bone Joint Surg Am 2011;93:1033-1038.

18. Swami VG, Cheng-Baron J, Hui C, Thompson RB, Jaremko JL. Reliability of 3D localisation of ACL attachments on MRI: Comparison using multi-planar 2D versus high-resolution 3D base sequences. Knee Surg Sports Traumatol Arthrosc 2015;23:1206-1214.

19. Davis DL, Chen L, Young ST. Evaluation of epiphyses in the skeletally immature knee using magnetic resonance imaging: A pilot study to analyze parameters for anterior cruciate ligament reconstruction. Am J Sports Med 2013;41: 1579-1585. 
20. Swami VG, Mabee M, Hui C, Jaremko JL. MRI anatomy of the tibial ACL attachment and proximal epiphysis in a large population of skeletally immature knees: Reference parameters for planning anatomic physeal-sparing ACL rreconstruction. Am J Sports Med 2014;42:1644-1651.

21. Lawrence JT, Bowers AL, Belding J, Cody SR, Ganley TJ. All-epiphyseal anterior cruciate ligament reconstruction in skeletally immature patients. Clin Orthop Relat Res 2010;468:1971-1977.

22. Harilainen A, Sandelin J. A prospective comparison of 3 hamstring ACL fixation devices-Rigidfix, BioScrew, and
Intrafix-randomized into 4 groups with 2 years of followup. Am J Sports Med 2009;37:699-706.

23. Kocher MS, Garg S, Micheli LJ. Physeal sparing reconstruction of the anterior cruciate ligament in skeletally immature prepubescent children and adolescents. J Bone Joint Surg Am 2005;87: 2371-2379.

24. Steiner ME, Hecker AT, Brown CH Jr, Hayes WC. Anterior cruciate ligament graft fixation: Comparison of hamstring and patellar tendon grafts. Am J Sports Med 1994;22(240):246. discussion 246-247. 